\title{
Contributors - Auteurs - Autoren
}

BAIN Jessica, Lecturer, Department of Media and Communication University of Leicester

Address: Room 2.05 Bankfield House, 132 New Walk, Leicester LE1 7JA, United Kingdom

Email: jb441@le.ac.uk

CHABAN Natalia, Jean Monnet Chair in European Identity and Culture and Deputy Director, National Centre for Research on Europe

Address: National Centre for Research on Europe, University of Canterbury, Kirkwood Village KD04, Private Bag 4800, Christchurch 8140, New Zealand Email: natalia.chaban@canterbury.ac.nz

CHRISTIE Sarah, Projects Coordinator, New Zealand European Union Centres Network

Address: c/o National Centre for Research on Europe, University of Canterbury, Kirkwood Village KD04, Private Bag 4800, Christchurch 8140, New Zealand

E-mail: sarah.christie@canterbury.ac.nz

CURLI Barbara, Associate Professor of Contemporary History, Jean Monnet Chair in History of European Integration, Università della Calabria Address: Dipartimento di Scienze politiche e sociali, via Pietro Bucci, Cubo 0B, 87036 Arcavacata di Rende (Cosenza), Italy

E-mail: barbara.curli@unical.it

DUCHENNE Geneviève, Visiting Professor, Université catholique de Louvain Address: Institut d'études européennes, Université catholique de Louvain, Place des Doyens 1, B-1348 Louvain-la-Neuve, Belgium

Email: genevieve.duchenne@uclouvain.be

GEHLER Michael, ad personam Jean Monnet Chair für europopäische Geschichte

Address: Institut für Geschichte, Stiftung Universität Hildesheim, Tilsiter Str. 1, D-31141 Hildesheim

Email: gehler@uni-hildesheim.de 
HOERBER Thomas, Associate Professor, Ecole Supérieure des Sciences Comemrciales d'Angers (ESSCA - School of Management), LUNAM University Adress: Centre for European Integration, 1 rue Lakanal, 49003 Angers, France E-mail: thomas.hoerber@essca.fr

MIOCHE Philippe, Professor of contemporary History, Aix-Marseille University

Address: Master Études européennes, Aix-Marseille Université (IUFM), 2 rue Jules Isaac, F-13626 Aix-en-Provence cedex 1

Email: philippe.mioche@univ-amu.fr

NASRA Skander, PhD Candidate, Ghent University

Address: Department of Political Sciences, Universiteitstraat 8, 9000 Ghent, Belgium Email: skander.nasra@ugent.be

SEGERS Mathieu, Assistant Professor, Utrecht University

Address: Department of History and Art History, Drift 10, 3512 BS Utrecht, The Netherlands

Email:m.1.1.segers@uu.nl

WILKENS Andreas, Professor of contemporary History, Université de Lorraine - Metz

Address: Département d'Histoire, UFR Sciences humaines et Arts, Ile du Saulcy, 57006 Metz cedex 1, France

Email: andreas.wilkens@univ-lorraine.fr 